\title{
Inhibition of tumor growth and tumor metastasis by a Chinese herbal formula - ZYD88, in an animal model with metastatic Lewis lung carcinoma
}

\author{
YAN-WEN DUAN ${ }^{1,2}$, YING HUANG ${ }^{1}$, LI-QUN CAI ${ }^{2}$, QUWEN DUAN ${ }^{1}$ and YUAN-SHAN ZHU ${ }^{1,2}$ \\ ${ }^{1}$ Changsha Cihang Research Institute of Materia Medica, Changsha, Hunan 410011, China; ${ }^{2}$ Department of \\ Medicine/Endocrinology, Weill Medical College of Cornell University, New York, NY 10021, USA
}

Received January 15, 2007; Accepted February 22, 2007

\begin{abstract}
Tumor metastasis is the leading cause of cancer death. In the present study, we determined the effectiveness of ZYD88, a Chinese herbal formula, in the inhibition of tumor growth and distant tumor metastases to the lung and liver in an animal model with metastatic Lewis lung carcinoma (LLC). Treatment with ZYD88 in adult C57BL/6 mice with metastatic LLC produced dose-dependent deceases in primary tumor weight, the mitotic tumor cell number, microvessel density, distant tumor metastases and red blood cell immune complexes, while it significantly increased tumor necrosis, thymus cortical thickness, the thymus medullar reticular epithelial cell (REC) number, and the activity of red blood cell $\mathrm{C} 3 \mathrm{~b}$ receptors. Although cyclophosphamide inhibited tumor growth, it had no significant effects on distant tumor metastases, thymus cortical thickness, the thymus medullar REC number, red blood cell $\mathrm{C} 3 \mathrm{~b}$ receptor activity and red blood cell immune complexes. These results indicate that ZYD88 possesses significant anti-angiogenic, anti-tumor and anti-metastatic effects in this animal model, and warrants further investigation.
\end{abstract}

\section{Introduction}

Tumor metastasis is the leading cause of death in cancer patients (1). It can occur early in tumorigenesis primary through two essential routes - the blood and lymphatic vessels. Although the genetic basis of tumorigenesis varies, the basic steps required for metastasis are similar for all tumors. Cancer cells metastasizing to distant sites from the primary

Correspondence to: Dr Yuan-Shan Zhu and Dr Yan-Wen Duan, Department of Medicine/Endocrinology, Weill Medical College of Cornell University, 1300 York Avenue, Box 149, New York, NY 10021, USA

E-mail: yuz2002@med.cornell.edu ywduan66@yahoo.com

Key words: lung cancer, herb, metastasis, angiogenesis tumor involve: Detaching from the primary tumor, invading the surrounding extracellular matrix, traveling in the blood or lymphatic vessels, attaching to the distant site, vessel formation or angiogenesis, and cell proliferation (2). It has been shown that $\sim 3$ million tumor cells can invade the circulation during a $24-\mathrm{h}$ period from each gram of an $\sim 8-\mathrm{g}$ tumor, but only $<0.1 \%$ of these cells are likely to survive and reach distant site (3). The complex processes involved in tumor metastasis can therefore become a burden, even though the malignant cells possess metastatic features. These multiple steps also provide various targets for anti-metastatic agents to block tumor metastasis (4), which can have significant implications for the treatment of cancer patients.

Chinese medicinal herbs, with a history that dates back thousands of years, have been used for the treatment of tumors for a long time. However, the efficacy and mechanisms of most of the Chinese herbal medicines remain to be evaluated. With the development of modern technology and the combination of western medicine, some progress has been made on the research and clinical application of Chinese medicinal herbs for cancer prevention and therapy (5-7). In the present study, we investigated the effectiveness of a Chinese medicinal herbal formula, ZYD88, derived from a traditional Chinese medicine, in the inhibition of tumor growth and distant tumor metastasis in an animal model with metastatic Lewis lung carcinoma (LLC). The results obtained suggest that ZYD88 was an effective agent for inhibiting tumor angiogenesis, tumor growth and distant tumor metastases in this LLC animal model. Furthermore, it improved the host's immunity with minimum side-effects, and warrants further investigation for the treatment of lung cancer, the most common cancer and the most common cause of cancer death in the world (8).

\section{Materials and methods}

ZYD88 preparation and chemicals. ZYD88 is composed of 6 Chinese medicinal herbs (Table I), and is processed by a proprietary technology that was developed by the Changsha Cihang Research Institute of Materia Medica (Changsha, China). Briefly, the entire processing of ZYD88 including such parameters as temperature, humidity, $\mathrm{pH}$, viscosity, pressure, extraction time, and yield, was consistently monitored by an automatic feedback system that consists of a central 
Table I. The Chinese herbal medicines components in ZYD88.

\begin{tabular}{lllr}
\hline $\begin{array}{l}\text { Name of Chinese } \\
\text { medicine (Pin Yin) }\end{array}$ & \multicolumn{1}{c}{ Latin name } & Part used & $\begin{array}{c}\text { Relative amount } \\
(\% \text { of total })\end{array}$ \\
\hline Da Huang & Radix et rhizoma rhei & Root & 10 \\
Ya Dan Zi & Fructus bruceae & Fruit & 10 \\
Huai Jiao & Fructus sophorae & Fruit & 30 \\
Yan Hu Suo & Rhizoma corydalidis & Tuber & 20 \\
Di Yu & Radix sanguisorbae & Root & 20 \\
Gan Cao & Radix glycyrrhizae & Root & 10 \\
\hline
\end{tabular}

control station with several peripheral sensors, regulators and quality control detectors. The presence of anthraquinone ingredients (emodin and rhein) and tetrahydropalmatine in each preparation was analyzed by thin-layer chromatography, using purified emodin, rhein and tetrahydropalmatine as the standards, respectively. The concentration of emodin in each preparation was determined by an absorbance at $510 \mathrm{~nm}$, and the average amount of emodin in 5 lots of ZYD88 analyzed was $0.622 \pm 0.056$ [standard deviation (SD)]\% (9). A liquid extract of ZYD88 at a concentration of $1 \mathrm{~g}$ of total raw herbs per $\mathrm{ml}$ was prepared and used for the experiments. The concentration was expressed as the weight of dry raw herbs in volume $(\mathrm{w} / \mathrm{v})$. Cyclophosphamide (CTX) was obtained from Shanghai 12th Pharmaceutical Co., Shanghai, China. RPMI-1640 medium was obtained from Invitrogen (Gibco, Cergy-Pontoise, France).

Animal model preparation and treatment. An animal model with highly metastatic LLC was prepared by inoculating tumor cells into the right flank of adult C57BL/6 mice as described previously with minor modifications (10). Briefly, Lewis lung tumor tissues dissected from xenograft animals (kindly provided by the Sichuan Antibiotic Research Institute, China) were washed once with RPMI-1640 medium, and then split up in 3 volumes of saline. Fifty-eight C57BL/6 mice (29 males and 29 females) at 9-11 weeks old were obtained from the Animal Center of West-China Medical University. These animals were injected subcutaneously in the right flank with $0.2 \mathrm{ml}$ tumor cell suspension, and randomly divided into 4 groups: Saline control $(\mathrm{n}=12)$, low-dose $(1.5 \mathrm{~g} / \mathrm{kg} / \mathrm{day}$, $\mathrm{n}=16)$ ZYD88, high-dose (6 g/kg/day, $\mathrm{n}=16)$ ZYD88, and the CTX group $(n=14)$. Each group consisted of half males and half females. ZYD88 treatment was started at the day of the tumor cell implantation, and continued for 20 days once a day (at 9:00-10:00) by gavage in $0.2 \mathrm{ml}$ volume. The same volume of saline was given to the control animals. CTX, used as the positive control, was given intraperitoneally (ip) at a dose of $8 \mathrm{mg} / \mathrm{kg}$ in $0.2 \mathrm{ml}$ every other day for 20 days. Similar doses of CTX and ZYD88 have been used in other animal models (11). The animals were housed in the Animal Center of West-China Medical University, China with a 12/12-h light/dark cycle, and had food and water ad libitum. All animal studies were approved by the Institutional Animal Care Committee, and conducted in accordance with accepted standards of humane animal care.
Tumor histology. At the end of the experiments, the animals were sacrificed and the primary tumor was dissected and weighed immediately. The percentage inhibition of tumor growth was calculated using the formula: Inhibition $(\%)=$ (average tumor weight in the control group - average tumor weight in the treated group)/average tumor weight in the control group $\mathrm{x} 100 \%$.

The dissected tumor tissues were fixed in $20 \%$ buffered formalin, embedded in paraffin, cut into $5-\mu \mathrm{m}$ thick sections and stained with H\&E according to standard histological procedures. Cell mitosis was examined under a microscope and identified morphologically in the H\&E-stained sections. The number of mitotic cells was counted under a microscope at $\mathrm{x} 400$ magnification, and the sum of the 5 highest fields was recorded for each specimen. The data are presented as the means $\pm \mathrm{SD}$. Tumor necrosis was measured as rare and involving $<0.5$ high-power field (HPF, x200) (+), frequent foci involving 0.5 to $1 \mathrm{HPF}(++)$ and confluent necrosis $>1 \mathrm{HPF}$ $(+++)$.

Analysis of tumor metastasis. Both the left and right lung from each animal were dissected and fixed by injecting $20 \%$ buffered formalin into the bronchi, then dehydrated, embedded in paraffin and cut into to $10-\mu \mathrm{m}$ sections. Three sections from each side of the lung were stained with H\&E and checked under a microscope for metastasis. The sum of metastatic foci in these six sections was recorded for each animal, and the data are presented as the means \pm SD in each group. The incidence of lung metastasis was calculated as the total number of animals with lung metastasis/total number of animals in the group $\mathrm{x} 100$.

In order to determine tumor metastasis to the liver, the liver was dissected and fixed in $20 \%$ buffered formalin. After dehydration, the tissues were paraffin-embedded, cut into 5- $\mu \mathrm{m}$ sections, and stained with H\&E. Tumor metastasis to the liver was examined in three sections, and scored as follows for each specimen: '+', Only one metastatic focus; '++', 2-4 metastatic foci; and ' +++ ', 5 or more metastatic foci in these sections.

Measurement of microvessel density. In order to assess the anti-angiogenic activity of ZYD88, paraffin-embedded tumor tissues were sectioned and stained with H\&E. The angiogenesis in each specimen was determined by counting the microvessel numbers. Microvessel counting was performed under a microscope at $\mathrm{x} 200$ magnification. The sum of the 5 
Table II. The inhibition of tumor growth by ZYD88 in an animal model with LLC.

\begin{tabular}{|c|c|c|c|c|c|c|c|}
\hline \multirow[t]{2}{*}{ Group } & \multirow{2}{*}{$\begin{array}{l}\text { No. of animals } \\
\quad \text { (start/end) }\end{array}$} & \multirow{2}{*}{$\begin{array}{l}\text { Tumor weight } \\
(\mathrm{g}, \text { mean } \pm \mathrm{SD})\end{array}$} & \multirow{2}{*}{$\begin{array}{l}\% \text { Tumor } \\
\text { inhibition }\end{array}$} & \multirow{2}{*}{$\begin{array}{l}\text { No. of mitotic cells } \\
\quad(\text { mean } \pm \text { SD) }\end{array}$} & \multicolumn{3}{|c|}{ Tumor necrosis } \\
\hline & & & & & + & ++ & +++ \\
\hline Control & $12 / 11$ & $3.81 \pm 0.98$ & & $38.36 \pm 6.78$ & 4 & 7 & 0 \\
\hline CTX & $14 / 14$ & $2.19 \pm 0.71^{\mathrm{a}}$ & 42.57 & $18.21 \pm 12.17^{a}$ & 0 & 1 & $13^{\mathrm{a}}$ \\
\hline ZYD88 (L) & $16 / 15$ & $2.46 \pm 0.74^{\mathrm{a}}$ & 35.34 & $9.92 \pm 5.69^{\mathrm{a}, \mathrm{b}}$ & 1 & 2 & $12^{\mathrm{a}}$ \\
\hline ZYD88 (H) & $16 / 15$ & $2.38 \pm 0.60^{\mathrm{a}}$ & 37.60 & $8.69 \pm 5.79^{a, b}$ & 0 & 0 & $15^{\mathrm{a}}$ \\
\hline
\end{tabular}

LLC, Lewis lung carcinoma; +, rare and involving <0.5 high-power field (HPF, x200); ++, frequent foci involving 0.5 to $1 \mathrm{HPF}$; +++, confluent necrosis >1 HPF; CTX, cyclophosphamide; L, low dose (1.5 g/kg/day); H, high dose (6 g/kg/day) ZYD88; ${ }^{\mathrm{a}} \mathrm{p}<0.01$ compared to the control group; ${ }^{\mathrm{b}} \mathrm{p}<0.05$ compared to the CTX group.

highest fields of vessel counts in three sections was recorded as the microvessel density for each specimen. The same number of fields was evaluated for each specimen. The values are presented as the means \pm SD for each experimental group.

Determination of the thickness of the cortical thymus and the number of reticular epithelial cells (REC) in the medullar thymus. The dissected thymuses were fixed in $20 \%$ buffered formalin, embedded in paraffin, cut into 5- $\mu \mathrm{m}$ sections, and stained with the Periodic Acid-Schiff (PAS) method. The thickness of the thymus cortex was measured under the microscope. The number of REC in the medullar thymus was counted and the sum of 5 high power fields (x400) was recorded.

Erythrocyte $C 3 b$ receptor rosette $(E-C 3 b R R)$ and erythrocyte immune complex rosette (E-ICR). The E-C3bRR and E-ICR were measured using red cell yeast mixture rose methods as previously described (12).

Statistic analysis. All quantitative data were presented as the means \pm SD. The difference between the control and treated groups was analyzed using an analysis of variance (ANOVA) following the post-hoc Fisher LSD test. The difference in proportions and ranked data was analyzed by Fisher's exact test. A p-value $<0.05$ was considered as statistically significant.

\section{Results}

ZYD88 inhibited tumor growth in the xenograft animal model with LLC. To determine the effects of ZYD88 on the inhibition of tumor growth, animals with LLC were treated with or without ZYD88 for 20 days. As shown in Table II and Fig. 1, treatment with ZYD88 at either a low dose $(1.5 \mathrm{~g} / \mathrm{kg} /$ day $)$, or a high dose $(6 \mathrm{~g} / \mathrm{kg} /$ day $)$ resulted in a significant decrease in primary tumor weight and size. As the positive control, treatment with CTX ( $8 \mathrm{mg} / \mathrm{kg}$ bw, ip, every other day), a cytotoxic chemotherapeutic agent, also inhibited tumor growth significantly. The effects of ZYD88 at both a low and a high dose on the inhibition of tumor growth were comparable with the dose of CTX used. No gender difference was observed in the ZYD88 inhibition of tumor growth.

ZYD88 treatment decreased cell proliferation as is evident by a decrease in the mitotic cell numbers in the tumor tissues as shown in Table II and Fig. 1 (compare Fig. 1B and C). Although CTX treatment also significantly decreased the mitotic cell numbers compared to the control, ZYD88 had a much greater effect than CTX. There were no significant differences between the low- and high-dose ZYD88 in the reduction of tumor weight and mitotic cell numbers.

Tumor necrosis was examined in order to determine the effects of ZYD88 on cell death. Treatment with either ZYD88 or CTX resulted in significant tumor necrosis in the xenograft tumors as shown in Table II and Fig. 1E, and an increase in fibrosis in the tumor tissues (Fig. 1E).

ZYD88 inhibited tumor metastasis to the lung and liver in the xenograft LLC animal model. As shown in Table III and Fig. 2A-D, ZYD88 produced significant decreases in the incidence of lung metastasis, and in the number of metastatic foci. Animals treated with either the low- or high-dose ZYD88 had a significantly lower incidence and decreased number of lung metastases compared to the control animals. In contrast, CTX failed to significantly inhibit tumor metastasis although it significantly inhibited tumor growth (Table III). Animals treated with high-dose ZYD88 tended to have much less lung metastatic foci and a lower incidence of metastasis than those animals treated with CTX although their anti-growth activity was comparable. However, the differences were not statistically significant ( $\mathrm{p}=0.07$ for foci, and $\mathrm{p}=0.08$ for incidence, respectively) due to the small sample sizes.

Animals with LLC also had significant liver metastasis, which was significantly inhibited by ZYD88 as shown in Table III and Fig. 2E-H. Compared to the control group, ZYD88 significantly decreased the incidence $(81.8 \%$ in the control group vs $13.3 \%$ in both the low- and high-dose ZYD88 groups) of tumor metastasis to the liver. In contrast, treatment with CTX did not significantly affect liver metastasis although it was decreased. Animals treated with ZYD88 at either the low- or high-dose had significantly less liver metastases when compared to the animals treated with CTX, (Table III) although they had a similar reduction in tumor weight (Table II).

ZYD88 decreased microvessel density in the xenograft LLC tumors. In order to evaluate the effects of ZYD88 on angiogenesis, we determined the microvessel density in the xenograft 

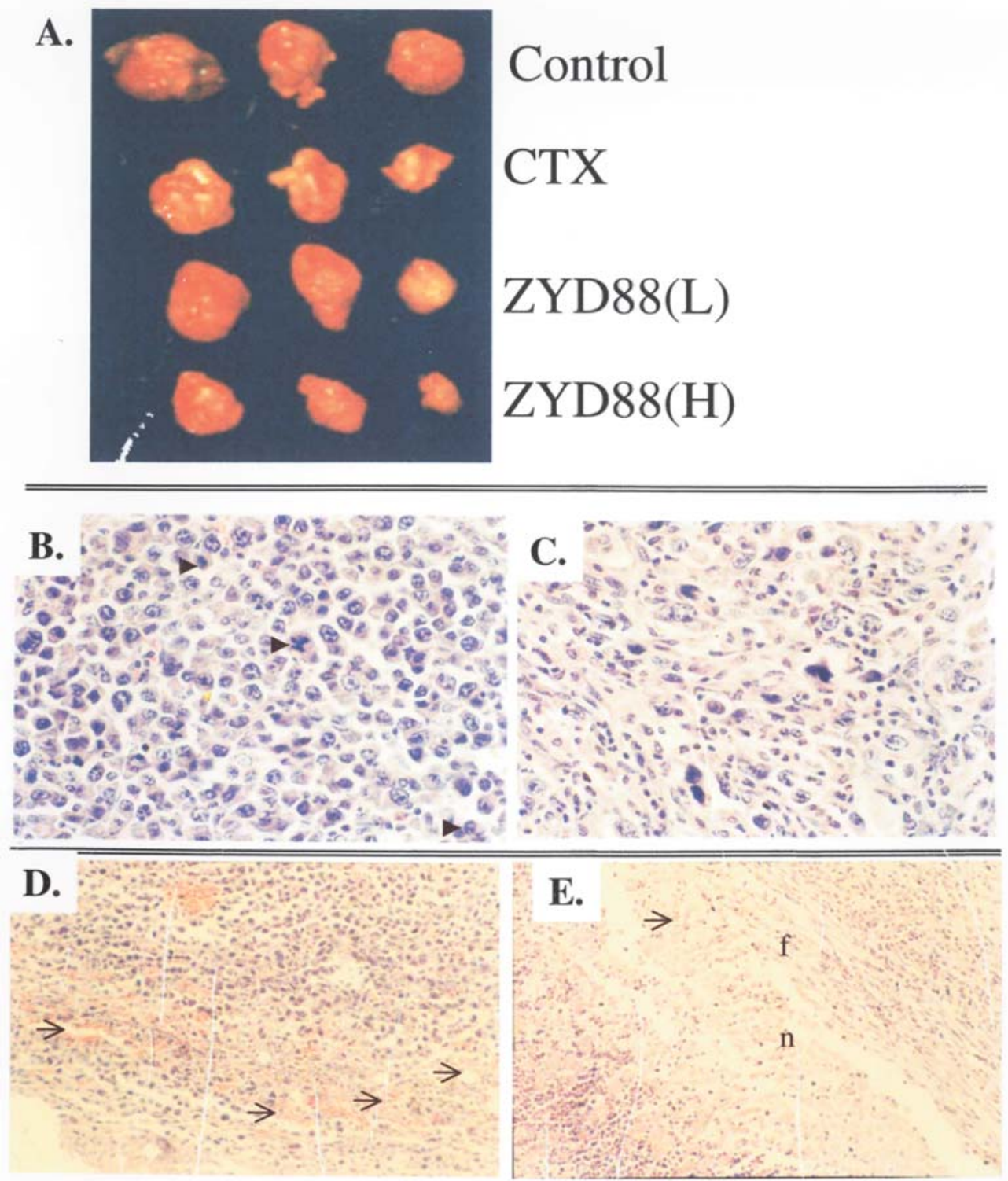

Figure 1. ZYD88 inhibited tumor growth and tumor cell proliferation while promoting cell death. (Panel A) Representative tumors dissected from animals treated with the vehicle control, cyclophosphamide (CTX), and the low- (L, $1.5 \mathrm{~g} / \mathrm{kg} / \mathrm{day})$, or high-dose ( $\mathrm{H}, 6 \mathrm{~g} / \mathrm{kg} / \mathrm{day})$ ZYD 88 . Three representative primary Lewis lung carcinoma (LLC) tumors from each group are photographed. (Panels B-E) Histological analysis of primary LLC tumors treated with or without ZYD88. The LLC tumor and histological analysis were carried out as described in 'Materials and methods'. Paraffin-embedded sections were stained with H\&E. Representative images of tumor tissues from the control (B and D) and ZYD88 (C and E) at a dose of $6 \mathrm{~g} / \mathrm{kg} / \mathrm{day}$ are presented. Tumor tissues from the control mice had large areas of confluent tumor cells with a large number of mitotic cells (arrow-heads), and normal surrounding microvessels (arrows). Tumors from the ZYD88-treated mice had few mitotic cells with more fibrosis (f), large areas of distinct necrosis (n) and occasional, isolated microvessels (arrows). The images in panels B and C are depicted at x400 magnification, and panels D and E, at x200 magnification.

Table III. The inhibition of tumor metastasis by ZYD88 in an animal model with LLC.

\begin{tabular}{|c|c|c|c|c|c|c|}
\hline \multirow[t]{2}{*}{ Group } & \multirow[t]{2}{*}{ No. of animals } & \multirow{2}{*}{$\begin{array}{l}\text { No. of lung metastatic } \\
\quad \text { foci }(\text { mean } \pm \text { SD) }\end{array}$} & \multirow{2}{*}{$\begin{array}{c}\text { Lung metastasis } \\
\text { (\% incidence) }\end{array}$} & \multicolumn{3}{|c|}{ Liver metastasis } \\
\hline & & & & - & + & ++ \\
\hline Control & 11 & $1.73 \pm 1.79$ & 63.6 & 2 & 7 & 2 \\
\hline CTX & 14 & $1.36 \pm 2.27$ & 35.7 & 6 & 6 & 2 \\
\hline ZYD88 (L) & 15 & $0.47 \pm 0.86^{\mathrm{a}}$ & $20.0^{\mathrm{b}}$ & $13^{\mathrm{b}, \mathrm{c}}$ & 2 & 0 \\
\hline ZYD88 (H) & 15 & $0.20 \pm 0.77^{\mathrm{a}}$ & $6.7^{\mathrm{a}}$ & $13^{\mathrm{b}, \mathrm{c}}$ & 2 & 0 \\
\hline
\end{tabular}

LLC, Lewis lung carcinoma; +, only one metastatic focus; ++, 2-4 metastatic foci; CTX, cyclophosphamide; L, low-dose (1.5 g/kg/day); H, high-dose (6 g/kg/day) ZYD88; ${ }^{\mathrm{a}} \mathrm{p}<0.01 ;{ }^{\mathrm{b}} \mathrm{p}<0.05$ compared to the control; ${ }^{\mathrm{c}} \mathrm{p}<0.05$ compared to the CTX group. 

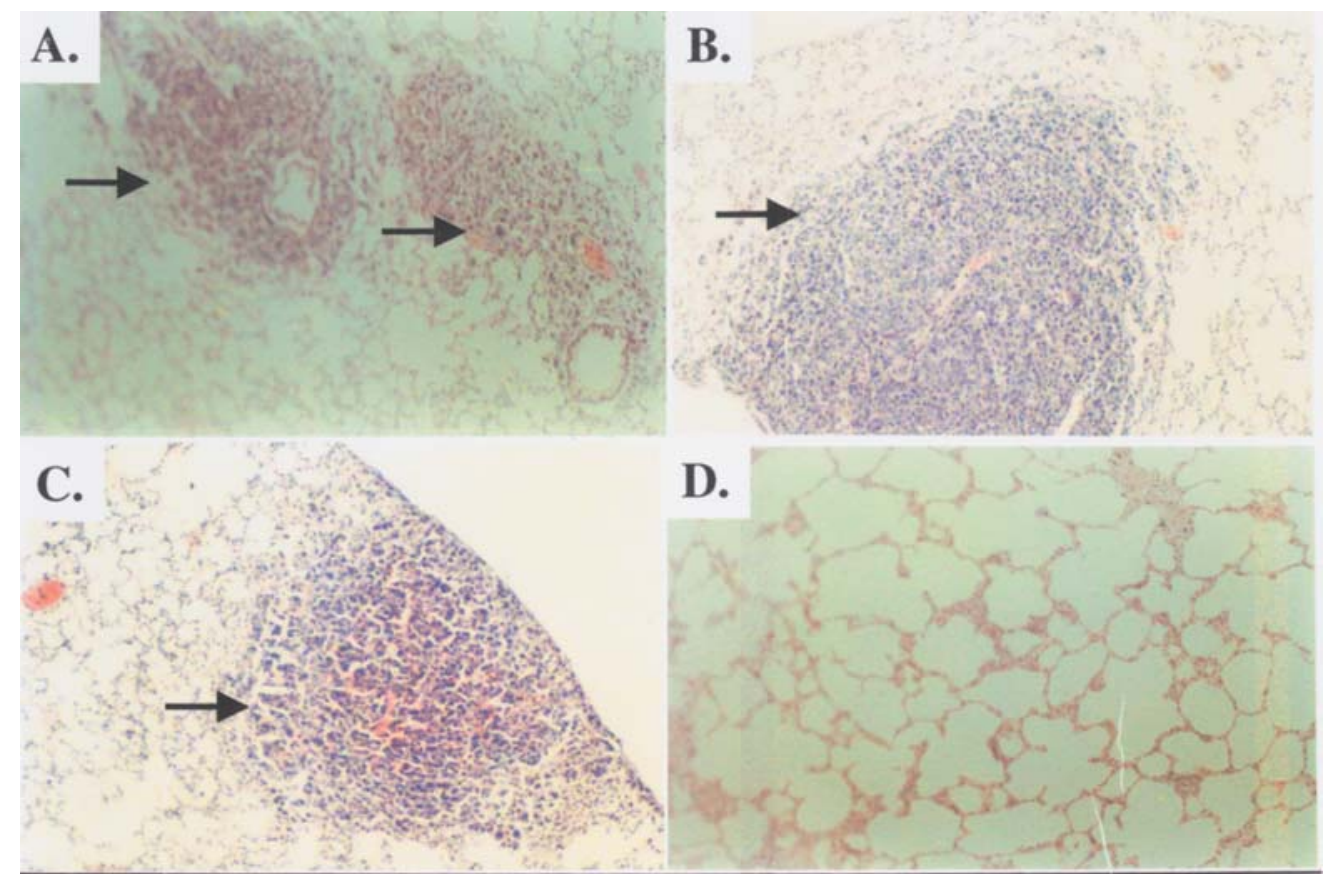

D.
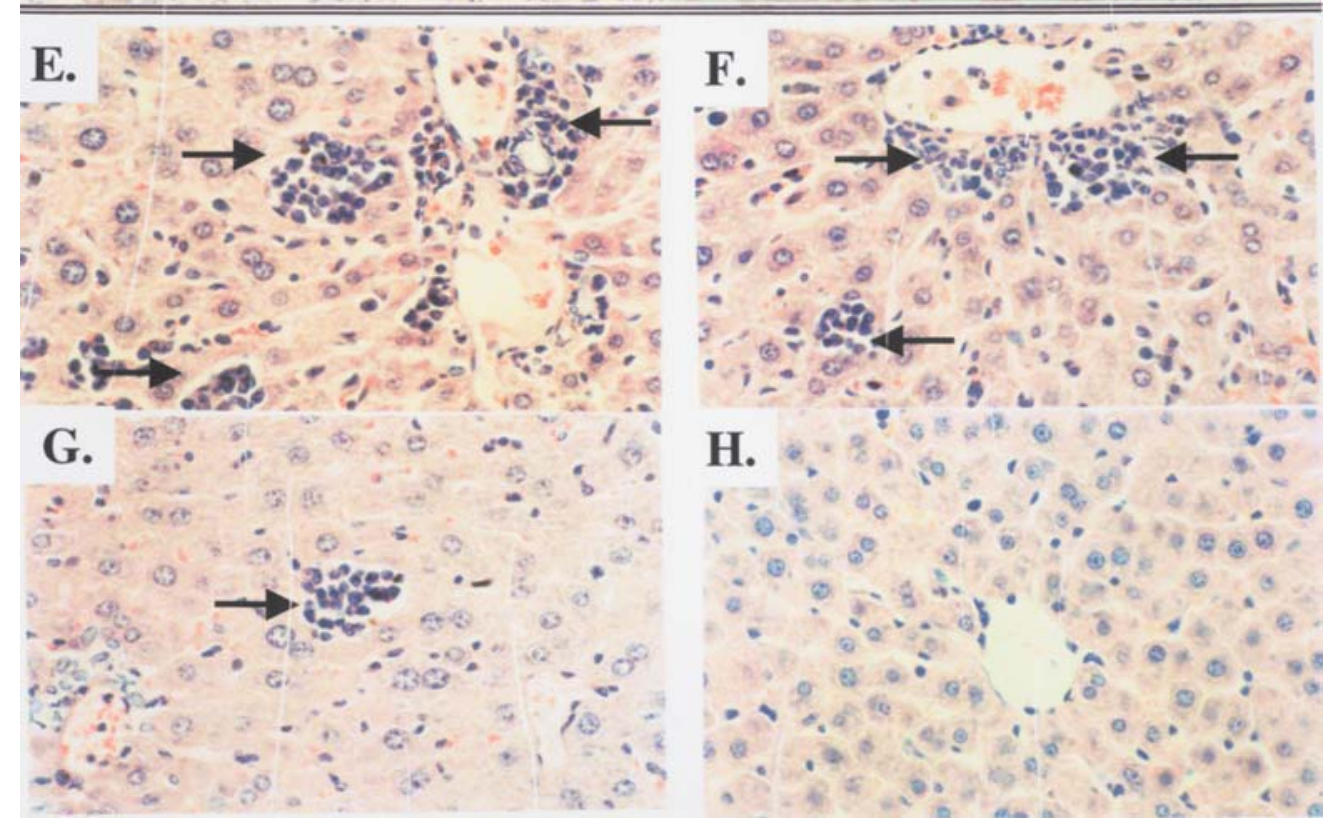

Figure 2. ZYD88 inhibited tumor metastasis to the lung (panels A-D) and liver (panels E-H). Representative images of metastatic lung tumor formation in animals treated with saline (A), cyclophosphamide (CTX) (B), low-dose (C) and high-dose (D) ZYD88, are presented. Treatment with ZYD88 produced a dose-dependent blockade of metastatic tumor formation in the lung. Panels E-H show the representative images of metastatic liver tumor formation in animals treated with saline $(\mathrm{E}), \mathrm{CTX}(\mathrm{F})$, and low- $(\mathrm{G})$ or high-dose $(\mathrm{H})$ ZYD88. Multiple and large metastatic foci pointed by arrows in the liver are observed in the control and CTX-treated animals, whereas only occasional, and focal metastatic foci are observed in the low-dose ZYD88-treated mice, and no metastatic foci in the high-dose ZYD88-treated animals (x200 magnification).

tumor tissues. As shown in Table IV and Fig. 1 (compare Fig. 1D and E), treatment with ZYD88 at either the low- or high-dose caused a significant decrease in microvessel numbers in the tumor tissues compared to both the control and CTX group. CTX also significantly decreased the microvessel density, but it was less effective than ZYD88.

Modulation of host immunity by ZYD88. In order to examine the effects of ZYD88 on host immunity, we determined the thickness of the thymus cortex, the REC numbers in the thymus medulla, the E-C3bRR and E-ICR. As shown in Table V, ZYD88 significantly increased the thickness of the thymus cortex, the number of REC in the thymus medulla, and the E-C3bRR, while it decreased the E-ICR compared to the control group. In contrast, CTX did not significantly affect the thymus and E-ICR although it slightly increased E-C3bRR. The ZYD88 effects on the thymus were significantly different from the CTX group.

\section{Discussion}

In the present study, we have demonstrated that ZYD88, a Chinese herbal medicine, produced a significant anti-tumor and anti-metastatic activity in a xenograft animal model with 
Table IV. The effect of ZYD88 on microvessel density in the LLC tissues.

\begin{tabular}{lcc}
\hline Group & No. of animals & $\begin{array}{c}\text { No. of blood } \\
\text { vessels } \\
\text { (mean } \pm \text { SD) }\end{array}$ \\
\hline Control & 11 & $45.7 \pm 26.7$ \\
CTX & 14 & $24 \pm 6.1^{\mathrm{a}}$ \\
ZYD88 (L) & 15 & $16.3 \pm 7.9^{\mathrm{a}, \mathrm{b}}$ \\
ZYD88 (H) & 15 & $12.2 \pm 5.6^{\mathrm{a}, \mathrm{b}}$ \\
\hline
\end{tabular}

LLC, Lewis lung carcinoma; CTX, cyclophosphamide; L, low-dose (1.5 g/kg/day); H, high-dose (6 g/kg/day) ZYD88; ${ }^{a} \mathrm{p}<0.01$ compared to the control group; ${ }^{\mathrm{b}} \mathrm{p}<0.01$ compared to the CTX $(8 \mathrm{mg} / \mathrm{kg}$, ip) group

LLC, a blood-born metastatic animal model (13). Treatment with ZYD88, at both a low and high dose, for 20 days beginning at the tumor cell inoculation, significantly inhibited the tumor growth as is evident by a decrease in tumor size and weight. This ZYD88 effect could be mediated through inhibiting tumor cell proliferation and promoting cell death since it decreased the numbers of mitotic cells, and increased cell necrosis in the tumor tissues (Table II and Fig. 1). The effects of ZYD88 on the inhibition of cell proliferation and promotion of cell death are further supported by our previous demonstration in cell cultures that ZYD88 inhibits cell proliferation and promotes cell apoptosis in SPC-A1 lung cancer cells (9), Lovo colon cancer cells (9), and multiple prostatic tumor cells (14).

Most importantly, the present study demonstrated that ZYD88 had a significant anti-metastatic activity as evidenced by the significant decreases in the numbers of metastatic foci and the incidence of metastasis to the lungs and liver in the LLC animal model. As CTX, a cytotoxic chemotherapeutic agent, produced a comparable inhibition of tumor growth as ZYD88 (Table II), but failed to inhibit tumor metastasis (Table III), the anti-metastatic effect of ZYD88 could involve some distinct mechanisms in addition to the inhibition of primary tumor growth. Two potential mechanisms accounting to this ZYD88 anti-metastatic activity could be related to its inhibition of angiogenesis and its promotion of host immunity. Numerous studies have indicated that angiogenesis plays a crucial role in tumor growth and tumor metastasis (15). An inhibition of angiogenesis by angiogenic inhibitors has been shown to decrease the blood supply to the tumor, resulting in an increase in cell death and a decrease in cell metastasis $(16,17)$. We have currently shown that ZYD88 significantly decreased microvessel density in the tumor tissues, which was much more effective than CTX even though they produced a comparable inhibition of tumor growth at the doses tested. Although the mechanisms behind the ZYD88 inhibition of angiogenesis remain to be elucidated, there is no doubt that its potent anti-angiogenic activity contributes, at least in part, to its anti-tumor and anti-metastatic effects.

It is well documented that alterations in host immunity are associated with tumor development and progression, and tumor cells are able to use multiple mechanisms to evade immune response and to repress host immunity $(18,19)$. Siegel and Gleicher reported that E-C3bRR is decreased, while E-ICR is increased in patients with cancer, suggesting that erythrocytes could be an important part of the immune system, and the immune-function is decreased in cancer patients $(20,21)$. Immunotherapy has been shown to inhibit tumor growth and tumor metastasis, and improve the outcome of cancer patients $(18,19)$. Our current experiments have shown that treatment with ZYD88 in LLC-bearing animals improved the host's immunity as demonstrated by the increases in the thymus cortical thickness, the thymus medullar REC numbers, and E-C3bRR, and a decrease in E-ICR. This feature of ZYD88 is a significant advantage over chemotherapeutic agents that usually have either no effect or deteriorative effects on the host immune system (22).

Since tumor metastasis involves multiple steps (2), the anti-metastatic activity of ZYD88 could also be mediated through other mechanisms. In addition to its anti-angiogenic activity and enhancement of host immunity, ZYD88 inhibits tumor cell proliferation, increases cell death (Table II and Fig. 1), and decreases tumor cell invasion to the surrounding tissues. Thus, the blockade of tumor metastasis by ZYD88 could be the outcome of ZYD88 actions on affecting multiple steps in the metastatic cascade.

Table V. The effect of ZYD88 on host immunity.

\begin{tabular}{lcccrr}
\hline Group & $\begin{array}{c}\text { No. of } \\
\text { animals }\end{array}$ & $\begin{array}{c}\text { Thickness of thymus } \\
\text { cortex }(\mathrm{mm}, \text { mean } \pm \text { SD) }\end{array}$ & $\begin{array}{c}\text { No. of REC } \\
(\text { mean } \pm \text { SD) }\end{array}$ & $\begin{array}{c}\text { E-C3bRR } \\
(\text { mean } \pm \text { SD) }\end{array}$ & $\begin{array}{c}\text { E-ICR } \\
(\text { mean } \pm \text { SD) }\end{array}$ \\
\hline Control & 11 & $0.129 \pm 0.061$ & $9 \pm 2$ & $9 \pm 1.27$ & $8.33 \pm 1$ \\
CTX & 14 & $0.159 \pm 0.040$ & $9.25 \pm 8.38$ & $11.25 \pm 2.56^{\mathrm{b}}$ & $7.20 \pm 2.04$ \\
ZYD88 (L) & 15 & $0.218 \pm 0.079^{\mathrm{a}, \mathrm{d}}$ & $22.36 \pm 9.14^{\mathrm{a}, \mathrm{c}}$ & $12 \pm 3.28^{\mathrm{a}}$ & $6 \pm 1.46^{\mathrm{a}}$ \\
ZYD88 (H) & 15 & $0.241 \pm 0.072^{\mathrm{a}, \mathrm{c}}$ & $28.31 \pm 7.59^{\mathrm{a}, \mathrm{c}}$ & $12.30 \pm 2.64^{\mathrm{a}}$ & $6.28 \pm 1.33^{\mathrm{a}}$ \\
\hline
\end{tabular}

REC, reticular epithelial cell; E-C3bRR, erythrocyte C3b receptor rosette; E-ICR, erythrocyte immune complex rosette; CTX, cyclophosphamide; L, low-dose (1.5 g/kg/day); H, high-dose (6 g/kg/day) ZYD88; ${ }^{\mathrm{a}} \mathrm{p}<0.01 ;{ }^{\mathrm{b}} \mathrm{p}<0.05$ compared to the control group; ${ }^{\mathrm{c}} \mathrm{p}<0.01$; ${ }^{\mathrm{d}} \mathrm{p}<0.05$ compared to the CTX $(8 \mathrm{mg} / \mathrm{kg}$, ip) group. 
In summary, our current study demonstrates that ZYD88, a Chinese herbal formula, significantly inhibits tumor growth and blocks tumor metastasis in the LLC animal model. Although the molecular basis and effective components of ZYD88 actions remain to be defined, multiple pathways could be involved since several components such as emodin, rhein (23), and genistein (24) in this Chinese herbal formula have been shown to possess anti-tumor and anti-metastatic activities via various cellular and molecular targets. As this herbal formula has minimum side-effects in animals and humans (11), it is a potential therapeutic agent for the treatment of lung cancer, and warrants further investigation for its clinical application.

\section{Acknowledgements}

This study was partially supported by the Key Technology R\&D Program of China (no. 96-903-01-10), the Innovation Fund For Small Technology-Based Firms of Ministry of Science and Technology of China (no. 99C26214300045), and the International Collaborative Items of State Administration of Foreign Experts Affairs (no. 20014300021).

\section{References}

1. Chambers AF, Groom AC and MacDonald IC: Dissemination and growth of cancer cells in metastatic sites. Nat Rev Cancer 2: 563-572, 2002.

2. Fidler IJ: The pathogenesis of cancer metastasis: the 'seed and soil' hypothesis revisited. Nat Rev Cancer 3: 453-458, 2003.

3. Liotta LA and Kohn E: Cancer invasion and metastases. JAMA 263: 1123-1126, 1990 .

4. Weiss L: Metastasis of cancer: a conceptual history from antiquity to the 1990s. Cancer Metastasis Rev 19: I-383, 2000.

5. Yu R: Taking the path of combining traditional Chinese medicine with western medicine in cancer research and control. Chin Med J 108: 732-733, 1995

6. Zheng S, Yang H, Zhang S, et al: Initial study on naturally occurring products from traditional Chinese herbs and vegetables for chemoprevention. J Cell Biochem S27: 106-112, 1997.

7. Niu H and Liu J: Advances in TCM treatment for metastasis of tumors. J Tradit Chin Med 23: 151-157, 2003.

8. Parkin DM, Bray F, Ferlay J and Pisani P: Global cancer statistics, 2002. CA Cancer J Clin 55: 74-108, 2005.

9. Duan Y, Huang Y, Duan Q and Zhu YS: Characterization of ZYD88, a Chinese herbal medicine, and its effects on tumor cell growth and colony formation in SPC-A1 lung and Lovo colon adenocarcinoma cells. Chin J Cancer Res (in press).
10. Kimura Y and Okuda H: Resveratrol isolated from Polygonum cuspidatum root prevents tumor growth and metastasis to lung and tumor-induced neovascularization in Lewis lung carcinomabearing mice. J Nutr 131: 1844-1849, 2001.

11. Huang Y, Duan Q, Zhu YS and Duan Y: In vivo antitumor activity of ZYD88, a Chinese herbal medicine in animal models with S180 sarcoma and Ehrlich tumor. J Soc Integrative Oncol (In press).

12. Guo F: Enhancement of the therapeutic effect and red cell immune function by radix Trichosanthis in mice bearing Ehrlich ascites carcinoma. Zhong Xi Yi Jie He Za Zhi 9: 418420, 1989.

13. Poggi A, Polentarutti N, Benedetta DM, de Gaetano G and Garattini S: Blood coagulation changes in mice bearing Lewis lung carcinoma, a metastasizing tumor. Cancer Res 37: 272-277, 1977.

14. Zhu YS, Huang Y, Cai LQ, et al: A Chinese medicinal herbal formula ZYD88 inhibits cell growth and promotes cell apoptosis in prostatic tumor cells. Oncol Rep 10: 1633-1639, 2003.

15. Folkman J: Role of angiogenesis in tumor growth and metastasis. Semin Oncol 29: 15-18, 2002.

16. O'Reilly MS, Holmgren L, Chen C and Folkman J: Angiostatin induces and sustains dormancy of human primary tumors in mice. Nat Med 2: 689-692, 1996.

17. Kuba K, Matsumoto K, Date K, Shimura H, Tanaka M and Nakamura T: HGF/NK4, a four-kringle antagonist of hepatocyte growth factor, is an angiogenesis inhibitor that suppresses tumor growth and metastasis in mice. Cancer Res 60: 6737-6743, 2000 .

18. Schirrmacher V, Umansky V and Rocha M: Immunotherapy of metastases. Curr Top Microbiol Immunol 213: 189-216, 1996.

19. O’Byrne KJ, Dalgleish AG, Browning MJ, Steward WP and Harris AL: The relationship between angiogenesis and the immune response in carcinogenesis and the progression of malignant disease. Eur J Cancer 36: 151-169, 2000.

20. Siegel I and Gleicher N: Red cell immune adherence (RCIA): its application in cancer and autoimmune disease. Immunol Commun 10: 433-449, 1981.

21. Currie MS, Vala M, Pisetsky DS, Greenberg CS, Crawford J and Cohen HJ: Correlation between erythrocyte $\mathrm{CR} 1$ reduction and other blood proteinase markers in patients with malignant and inflammatory disorders. Blood 75: 1699-1704, 1990.

22. Hadden JW: Immunodeficiency and cancer: prospects for correction. Int Immunopharmacol 3: 1061-1071, 2003.

23. Shi YQ, Fukai T, Sakagami H, et al: Cytotoxic and DNA damage-inducing activities of low molecular weight phenols from rhubarb. Anticancer Res 21: 2847-2853, 2001.

24. Wietrzyk J, Opolski A, Madej J and Radzikowski C: Antitumour and antimetastatic effect of genistein alone or combined with cyclophosphamide in mice transplanted with various tumours depends on the route of tumour transplantation. In Vivo 14: 357-362, 2000. 